\title{
O projecto de pontes para vidas superiores a 100 anos
}

\author{
F. A. Branco ${ }^{1}$, P. V. Paulo ${ }^{1}$
}

${ }^{1}$ IST-ICIST Universidade Técnica de Lisboa. Av. Rovisco Pais. Lisboa, Portugal.

\section{Información del artículo \\ DOI: \\ http://dx.doi.org/10.21041/ra.v2 \\ $\underline{i 1.20}$ \\ Artículo recibido el 10 de \\ Octubre de 2011, revisado bajo \\ las politicas de publicación de \\ la Revista ALCONPAT y \\ aceptado el 08 de Diciembre de \\ 2011. Cualquier discusión, \\ incluyendo la réplica de los autores se publicará en el tercer número del año 2012 siempre y cuando la información se reciba antes del cierre del segundo número del año 2012}

\section{(C) 2012 Alconpat Internacional}

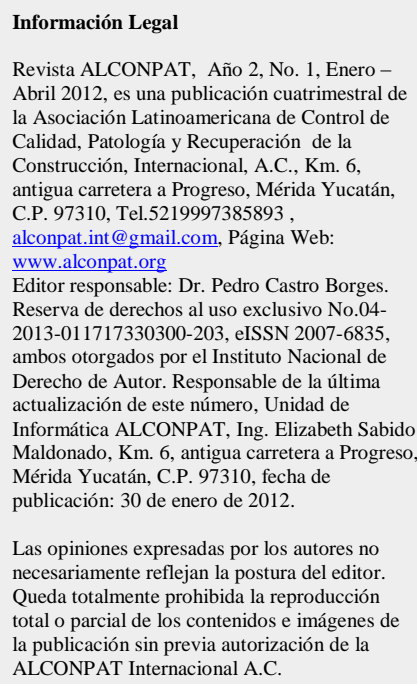

\section{RESUMO}

Apresenta-se uma nova filosofia de concepção das pontes para mais de 100 anos de vida útil que contempla de forma integrada as várias fases desde o projecto, passando pela construção e incluindo a fase de serviço, tendo em vista atingir a durabilidade pretendida. A metodologia apresentada é ilustrada com a experiência obtida com a Ponte Vasco da Gama que foi a primeira ponte Portuguesa onde foi imposta uma vida útil de 120 anos.

Palavras chave: pontes; vida útil; durabilidade; projecto; gestão.

\section{ABSTRACT}

A new philosophy for bridge design is presented for structures with more than 100 years of service life. It considers in an integrated way the design phase with special structural analysis and a durability study, the construction phase with high quality control and the service phase with monitoring and inspection within a management system. This methodology is illustrated with de design of the Vasco da Gama bridge which was the first Portuguese bridge to be designed for 120 years service life.

Keywords: bridges; service life; durability; design; management.

Autor de contacto: Fernando Branco (fbranco@ civil.ist.utl.pt) 


\section{DISCUSSÃO}

Numa altura em que se começam a projectar as grandes pontes para mais de 100 anos de vida útil, é necessário adoptar uma nova filosofia de concepção das pontes que contemple, de forma integrada, as várias fases da obra desde o projecto até à fase de serviço, em termos de durabilidade. O processo inicia-se com o desenvolvimento de um projecto de durabilidade, a que se segue um conjunto de procedimentos durante a construção para garantir as especificações de projecto. Durante a fase de serviço, a gestão da ponte é realizada incorporando um sistema de inspecção e manutenção para controlo das degradações. A metodologia apresentada é ilustrada com a experiência adquirida na Ponte Vasco da Gama que foi a primeira ponte Portuguesa onde foi imposto, na fase de concurso, a realização de um projecto para uma vida útil de 120 anos.

\section{INTRODUÇÃO}

A importância da degradação das pontes está a gerar grande preocupação nos organismos governamentais, já que os custos envolvidos na manutenção e na quebra de funcionalidade das pontes existentes são cada vez maiores. As razões da degradação resultam basicamente de um deficiente projecto, da falta de controlo de qualidade durante a construção e ainda da falta de realização de inspecções e de manutenção periódicas durante a fase de serviço.

Numa altura em que se começam a projectar as grandes pontes para mais de 100 anos de vida útil, é necessário adoptar uma nova filosofia de concepção das pontes que contemple, de forma integrada, as várias fases da obra desde o projecto até à fase de serviço, em termos de durabilidade. A metodologia global apresentada é ilustrada com a experiência adquirida com a Ponte Vasco da Gama que foi a primeira ponte Portuguesa onde foi imposto, na fase de concurso, a realização de um projecto para uma vida útil de 120 anos (Branco, 1999) (Figura1).

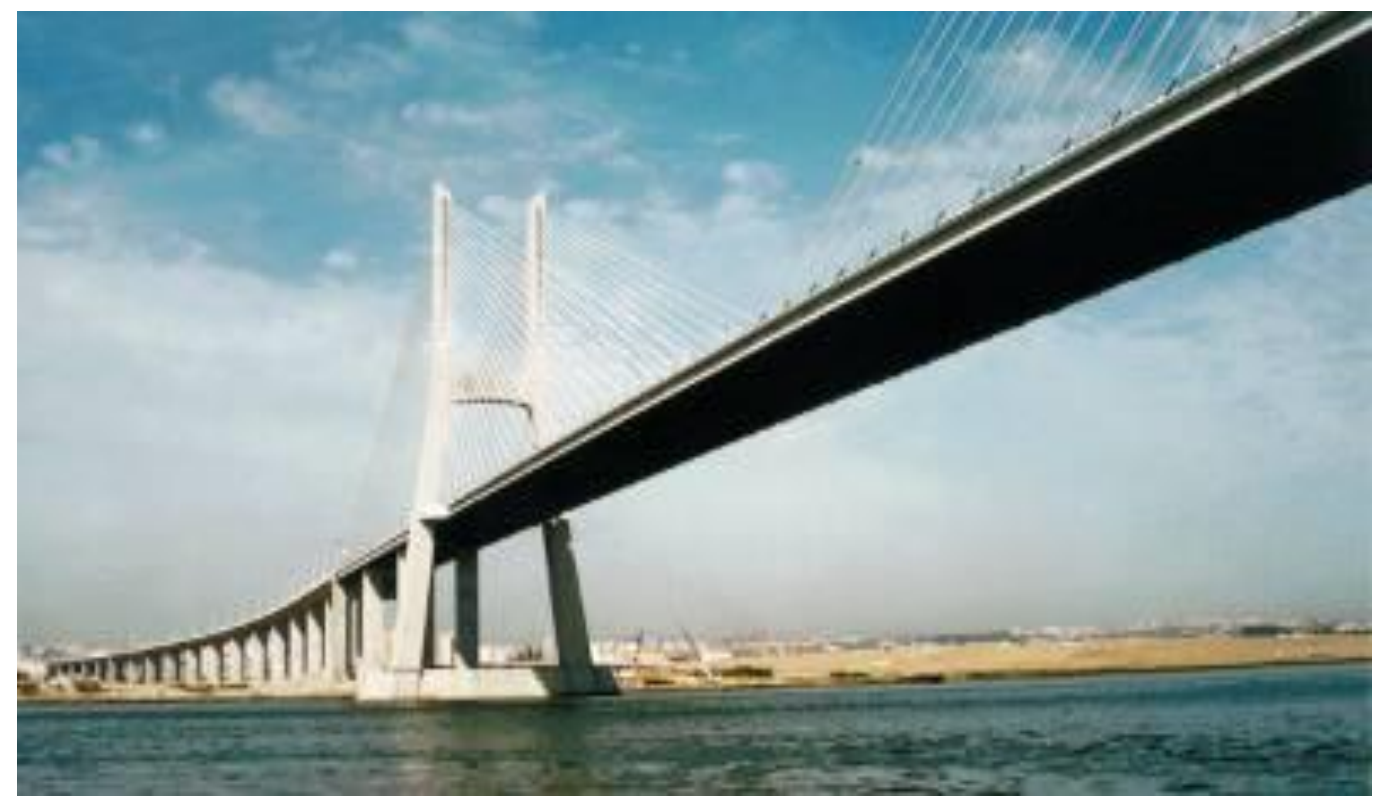

Figura 1. Ponte Vasco da Gama projectada para uma vida útil de 120 anos 


\section{A FASE DE PROJECTO}

\subsection{Definição da Vida Funcional da Obra}

O principal objectivo da construção de uma ponte é melhorar a circulação rodoviária da travessia. Por isso a caracterização da vida de uma ponte tem de começar pela definição da sua vida funcional ou seja pela caracterização da máxima capacidade de tráfico pretendida. Com base nas previsões iniciais da evolução do tráfico é definido à partida o número de vias do tabuleiro da ponte que suportarão, em boas condições de funcionalidade, o tráfico durante a vida "funcional" da ponte (TRB, 1995). Esta vida funcional é frequentemente inferior à vida estrutural da ponte, o que significa que a partir de certa altura começarão a ocorrer os problemas de congestionamento rodoviário. Nesta situação a ponte deverá ser reabilitada funcionalmente, por exemplo aumentando a sua largura ou construindo uma nova ponte perto desta, de modo a garantir a manutenção da qualidade funcional da travessia.

\subsection{Definição da Vida Estrutural da Obra}

Os Códigos de projecto estão habitualmente concebidos para construções com vidas de 50-60 anos. Para as pontes importantes, em que se começam a impor vidas úteis maiores (100-120 anos), não há habitualmente regulamentos aplicáveis. Tal implica assim não só adaptar os actuais regulamentos de segurança estrutural, como adoptar modelos especiais de degradação par analisar os problemas de durabilidade.

Nestas situações, tem também de se definir o significado de limite da vida. Frequentemente adopta-se, um fim da vida útil, associado ao início da corrosão nas armaduras principais de betão armado ou associado ao início da corrosão nas secções úteis de aço das estruturas metálicas.

\subsection{O Projecto de Segurança Estrutural}

Em termos de segurança estrutural os Códigos Estruturais (ENV, 1991 - 1999) definem os valores estatísticos (característicos) das Acções $\left(A_{k}\right)$ e da Resistência dos Materiais $\left(R_{k}\right)$ que devem ser usados em projecto de modo a garantir um elevado nível de segurança estrutural $\left(\mathrm{P}\left(\mathrm{A}_{\mathrm{k}}\right)>\mathrm{R}_{\mathrm{k}}<7 \times 10^{-5}\right)$, sendo esta segurança associada ao período de referência da vida útil (habitualmente cerca de 50 anos).

Se a vida estrutural for superior a 50 anos, o valor das acções variáveis tem também de ser superior, de modo a garantir-se para esse período o mesmo nível de segurança. Estes valores de correcção estão hoje definidos nos Códigos (para vento e sismos ver ENV-1991 1999 e ENV1998 1995) e devem ser adoptados no projecto de pontes para vidas de 100-120 anos

No projecto de segurança estrutural de pontes importantes, com elevados tempos de vida útil devem ainda ser considerados os efeitos de acções especiais (frequentemente não referidos nos Códigos para as pontes correntes), tais como sobrecargas anormais de veículos, situações de acidente, choque de navios, etc. 


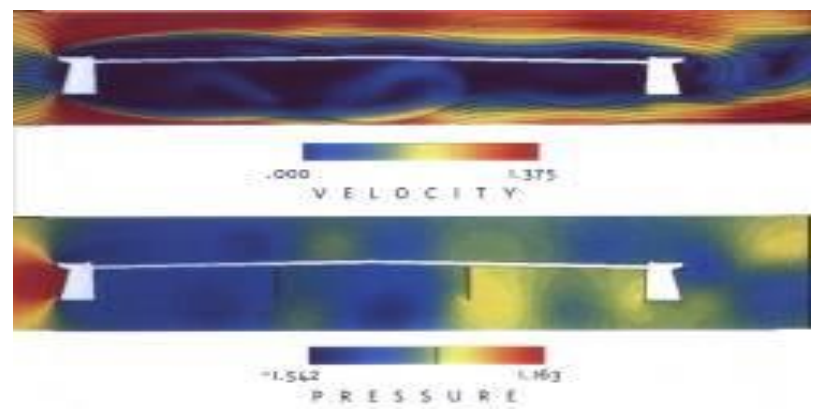

Figura 2. Estudos aerodinâmicos

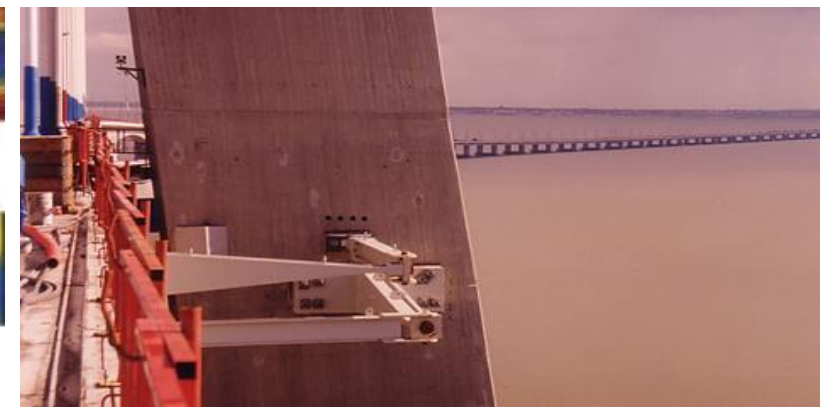

Figura 3. Amortecedores do tabuleiro

Com exemplo referem-se alguns destes estudos especiais de segurança estrutural realizados para a Ponte Vasco da Gama. Os estudos do seu comportamento aerodinâmico foram feitos em túnel de vento para analisar a sua estabilidade para rajadas de $250 \mathrm{~km} / \mathrm{h}$. A geometria inicial da secção transversal conduzia a instabilidade para velocidades a partir de $170 \mathrm{~km} / \mathrm{h}$ e através numa análise inovadora com CFD (computer fluid dynamics) e de testes adicionais de túnel, obteve-se uma solução estável com a colocação de protecções sob o tabuleiro (Figura 2). Nesta obra foram também realizadas análises sísmicas detalhadas associadas ao período de referência de 120 anos. Foi ainda desenvolvido um estudo sísmico específico para analisar as situações de liquefacção das fundações e para definir as características dos amortecedores colocados entre o tabuleiro e as torres, reduzindo os deslocamentos do tabuleiro (Figura 3) (Branco, 2000).

Em termos de acções especiais, as bases das torres principais foram analisadas para o embate acidental de um navio com 30 000ton (DWT) à velocidade de 10 nós e foi feito o estudo da situação de acidente associada a um incêndio de um navio sob o tabuleiro (Branco, 2000).

\subsection{Projecto de Durabilidade}

O projecto de durabilidade para uma ponte em que se pretende atingir uma vida útil de 120 anos, passa pela análise dos aspectos a seguir descritos e que deve ser realizado em paralelo ao projecto de segurança estrutural, acima referido.

3.4.1 Definição das propriedades dos materiais. A estimativa da vida útil das pontes com base na deterioração dos materiais é um problema complexo, sujeito a grande investigação em muitos países do mundo (Branco, 2004). Essa estimativa inclui a caracterização do ambiente e a definição de modelos matemáticos para simular as degradações.

Nas pontes em betão armado, os principais mecanismos de degradação estão associados às situações de carbonatação e ao ataque de cloretos em ambientes salinos. Nas estruturas correntes, com vidas úteis da ordem dos 50-60 anos, as Normas Europeias (NP EN-206 2007) definem ambientes para cada mecanismo de degradação, recomendações para os betões a adoptar (quantidade de cimento, relação água/cimento, resistência) e recobrimentos de armaduras.

Para as pontes importantes, com vidas úteis maiores (100-120 anos), não há regulamentos aplicáveis. Aqui o estudo da vida útil tem de ser realizado com base na deterioração dos materiais, nas condições ambientais e nas condições limite definidas, usando modelos matemáticos para a deterioração (Branco, 2004). Estes modelos têm também vindo a ser desenvolvidos essencialmente para a carbonatação e para o ataque dos cloretos.

A carbonatação do betão resulta da penetração do $\mathrm{CO}_{2}$ nos poros do betão e desenvolve-se numa frente cuja profundidade "d" pode ser estimada, ao longo do tempo "t" (anos), por 


$$
\mathrm{d}=\mathrm{K}(\mathrm{t})^{0.5}
$$

onde K é o coeficiente de carbonatação, com valores variáveis entre 1 e 8 (mm/ano $\left.{ }^{0,5}\right)$ em função da permeabilidade do betão e das condições ambientais, e valores médios de de $3,5 \mathrm{~mm} /$ ano $^{0,5}$ (Monteiro, 2010).

A penetração dos iões cloro no betão pode ser simulada pela lei de difusão de Fick. A solução da equação diferencial, tendo em conta a variação com o tempo, do coeficiente de difusão dos cloretos $\mathrm{D}_{\mathrm{c}}\left(\mathrm{cm}^{2} / \mathrm{sec}\right)$, permite obter a concentração de cloretos $\mathrm{C}(\mathrm{x}, \mathrm{t})$ dentro do betão à profundidade $\mathrm{x}(\mathrm{cm})$ e tempo $\mathrm{t}(\mathrm{sec})$, por

$$
\mathrm{C}(\mathrm{x}, \mathrm{t})=\mathrm{C}_{\mathrm{o}}\left(1-\operatorname{erf}\left(\mathrm{x} /\left(2\left(\mathrm{D}_{\mathrm{c}} \mathrm{t}^{(1-\mathrm{m})} /(1-\mathrm{m})\right)^{0.5}\right)\right)\right.
$$

onde $\mathrm{C}_{\mathrm{o}}$ é a concentração de cloretos à superfície (\% do peso de cimento), "erf" é a função erro e "m" uma constante empírica $(\mathrm{m}=0,4)$ (Mangat, 1994).

Com estes modelos de carbonatação e de ataque de cloretos pode-se estimar a evolução da degradação da estrutura e definir especificações para os materiais, nomeadamente em termos das propriedades do betão e do recobrimento das armaduras.

Este tipo de análises foi aplicado à Ponte Vasco da Gama onde as estruturas foram divididas em várias zonas ambientais (submersa; entre-maré; zona de salpicos; humidade elevada, humidade média) e para cada uma delas foram quantificados os recobrimentos a adoptar e as características de permeabilidade dos betões (Tabela 1).

Tabela 1. Características do betão para uma vida útil de 120 anos em ambiente salino

\begin{tabular}{|c|c|c|c|}
\hline Structure & $\begin{array}{c}\text { Coef. Difusão } \\
\text { (x 10 -12 m2/s aos 28 dias) }\end{array}$ & $\begin{array}{c}\text { AASHTO } \\
\text { Teste (Coulombs) }\end{array}$ & $\begin{array}{c}\text { Recob. } \\
\text { (mm) }\end{array}$ \\
\hline V.Gama (imersão) & 5 & 3000 & 70 \\
V. Gama (salpicos) & 2 & 1500 & 70 \\
V. Gama (ar) & 5 & 3000 & 50 \\
\hline
\end{tabular}

No que se refere às estruturas metálicas a vida útil pode ser estimada com base em taxas de corrosão do aço. Aqui deve ser adoptada uma sobre-espessura que considere a parte que vai ser corroída durante a vida útil. A tabela 2 apresenta taxas de corrosão em ambientes marítimos, para aço não protegido, que permitem estimar sobreespessuras para valores definidos de vida útil.

Tabela 2. Taxas de corrosão em aço para ambientes marítimos

\begin{tabular}{|l|c|}
\hline \multicolumn{1}{|c|}{ Localização } & $\boldsymbol{\mu m} / \mathbf{a n o}$ \\
\hline Zona enterrada & 0,01 \\
Submersa em lodos & 0,1 \\
Totalmente submersa & $0,08-0,12$ \\
Zona inter-maré & $0,1-0,2$ \\
Zona de salpicos & $0,2-0,4$ \\
Exposição ao ar & $0,1-0,2$ \\
\hline
\end{tabular}

3.4.2 Concepção com flexibilidade. A vida útil definida em projecto está associada aos principais elementos estruturais, considerando uma manutenção corrente. No entanto há componentes da 
ponte com uma vida útil inferior à vida útil global, o que levará a obras de reparação/substituição durante a fase de serviço da obra (apoios, juntas, etc. são elementos que tipicamente têm vidas mais curtas). A análise da durabilidade destes elementos deverá também ser feita na fase de projecto.

Como exemplo, na Ponte Vasco da Gama foi considerada uma solução em que as juntas suportam apenas acções sísmicas do nível de traficabilidade (75\%), colapsando para sismos superiores. O sistema flexível conduziu a juntas menores e portanto com menor manutenção.

3.4.3 Monitorização e fácil inspecção. As pontes importantes deverão ser monitorizadas para se acompanhar o seu comportamento estrutural e de durabilidade ao longo da fase de serviço. Todas estas actividades de monitorização têm de ser pensadas na fase de projecto, deixando na obra os respectivos equipamentos de monitorização, com transmissão de dados para uma central de tratamento. $\mathrm{O}$ acesso a estes equipamentos tem também de ser pensado desde a fase de projecto com soluções com fácil acesso para os inspectores que visitarão a obra. A Ponte Vasco da Gama foi concebida com carros de inspecção do tabuleiro e acessos às zonas a inspeccionar (Figura 4).

3.4.4 Especificações técnicas e plano base de manutenção. Os aspectos atrás referidos sobre durabilidade devem ser apresentados em especificações técnicas (especificações de durabilidade) para os materiais e componentes da ponte, incluindo a definição dos ensaios a realizar, e os parâmetros de durabilidade a atingir. Tendo em conta a vida útil estimada para a ponte, deverá também ser elaborado um plano base de inspecções periódicas a ser complementado no início da fase de serviço, aspectos considerados na Ponte Vasco da Gama.

\section{CONSTRUÇÃO COM DURABILIDADE}

Durante a fase de construção, um bom controlo de qualidade é o melhor processo para se garantir a vida útil definida em projecto. Nesta fase as principais actividades de controlo da qualidade, relacionadas com a durabilidade são as que a seguir se descrevem.

\subsection{Caracterização inicial dos materiais}

Antes de se iniciarem as betonagens o empreiteiro deverá estudar as composições do betão de modo a atingir as características especificadas para os materiais em termos de durabilidade. Isto é particularmente importante porque os ensaios de durabilidade mais fiáveis são demorados, pelo que devem ser realizados o mais cedo possível.

Na Ponte Vasco da Gama o estudo inicial da durabilidade dos betões aos cloretos foi realizada usando inicialmente os ensaios AASHTO T270 e de Tang Lupping (Mangat, 1994). Posteriormente todos os betões foram também analisados com ensaios (demorados) de permeabilidade aos cloretos (Branco, 1999).

\subsection{Controlo in situ das propriedades dos materiais}

$\mathrm{O}$ controle dos materiais deve também ser feito in-situ de modo a verificar-se se as características de durabilidade obtidas em laboratório se mantêm. Este controle periódico é fundamental para a qualidade da obra final.

Na Ponte Vasco da Gama, entre os parâmetros de durabilidade medidos in situ consideraram-se o coeficiente de difusão e a concentração superficial dos cloretos que foram utilizados para calibrar os modelos adoptados em projecto para previsão da vida útil. Foram também medidos in-situ e 
em laboratório parâmetros mecânicos como a resistência à compressão, módulo de elasticidade, fluência e retracção, parâmetros importantes para analisar o comportamento ao longo do tempo da ponte, nomeadamente associados às deformações do tabuleiro.

\subsection{Métodos de construção}

Os métodos de construção devem ser implementados de modo a garantir os melhores procedimentos para se atingir uma boa durabilidade, nomeadamente no controle da execução da camada superficial do betão dos elementos estruturais, ou seja no recobrimento (Branco, 1999). As actividades de vibração, cura, controle de calor de hidratação, etc, destas zonas superficiais, são muito importantes para obter uma boa durabilidade.

Na Ponte Vasco da Gama, como exemplo, referem-se dois problemas construtivos ocorridos que poderiam afectar a durabilidade da obra. O primeiro resultou da fissuração superficial dos grandes recobrimentos $(7 \mathrm{~cm})$, associada a retracção plástica da zona não armada, e a uma redução da protecção das armaduras. Tal foi solucionado com curas cuidadas e com a injecção das zonas fissuradas. $\mathrm{O}$ outro problema resultou de recobrimentos deficientes em pilares estacas, resultante da não verticalidade das armaduras. A sua correcção conduziu à realização de protecções em fibra de vidro, para garantir a vida útil contratual de 120 anos (Figura 5).

\subsection{Ensaios de carga e de recepção}

A realização de ensaios de carga no fim da obra é um aspecto importante para verificar a fiabilidade dos modelos de cálculo utilizados na fase de projecto e para definir um estado de referência para o resto da vida da obra.

Os ensaios realizados na Ponte Vasco da Gama com cargas estáticas permitiram comprovar as flechas e as extensões que ocorrem sob o efeito das sobrecargas. Os ensaios dinâmicos permitiram obter as características dinâmicas da ponte e as forças nos tirantes, calculadas através das suas frequências próprias.

\section{GESTÃO DURANTE A VIDA ÚTIL}

Para garantir o bom comportamento da estrutura durante a vida útil de uma ponte importante tem de existir um sistema de monitorização permanente das variáveis mais significativas, além de serem implementados procedimentos correntes e periódicos de inspecção e manutenção. De modo a racionalizar estas actuações (em termos técnicos e de custos), os procedimentos devem estar incluídos em sistemas de gestão de pontes cujas características principais são brevemente apresentadas.

\subsection{Monitorização Estrutural}

A monitorização estrutural destina-se controlar em permanência os principais parâmetros associados ao comportamento estrutural da ponte de modo a detectar instantaneamente eventuais problemas estruturais decorrentes de anomalias ou acidentes.

$\mathrm{Na}$ Ponte Vasco da Gama é feita a medição automática de deslocamentos, rotações, extensões, temperaturas e vibrações em secções pré-definidas e existe ainda um sistema independente de medição de acelerações sísmicas. Estas medições estão associadas a um plano de acções onde em função dos valores medidos e de níveis pré-definidos em projecto, se implementam decisões rápidas em caso de acidente, sismo ou vento excessivo. 


\subsection{Monitorização de durabilidade}

O plano de monitorização da durabilidade define o ensaio periódico dos materiais necessários à reavaliação da evolução da degradação dos materiais durante a vida útil. Se a deterioração for superior à prevista em projecto, tal permitirá implementar medidas atempadas de reparação. É esta monitorização que permite quantificar os coeficientes reais da obra/ambiente associados a $\mathrm{K}$ (carbonatação) e $\mathrm{D}_{\mathrm{c}}$ (cloretos) e assim saber-se com bastante rigor a evolução da degradação.

$\mathrm{Na}$ Ponte Vasco da Gama, os ensaios de controlo foram realizados a 0,5, 1, 2, 5 e todos os 5 anos depois da conclusão da construção. Em relação aos elementos de betão é medida a carbonatação, o perfil de cloretos, o coeficiente de difusão, a permeabilidade, a porosidade e os potenciais eléctricos. Em relação aos elementos metálicos (estacas metálicas) a medição da sua espessura é medida debaixo de água com ultra sons, cada 2 anos.

Os resultados das campanhas de monitorização da durabilidade durante os primeiros anos mostraram que até agora os níveis de carbonatação e propagação de cloretos estão dentro do previsto na fase de projecto. Houve no entanto problemas associados à existência de reduzidos recobrimentos que já levaram à implementação de medidas de protecção adicional (Figura 5).

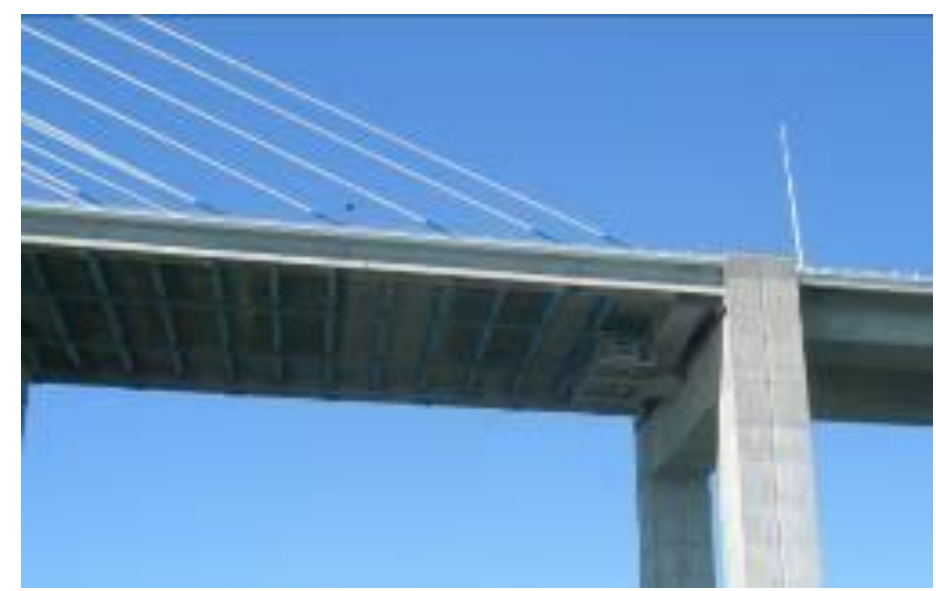

Figura 4. Veículo de inspecção da ponte

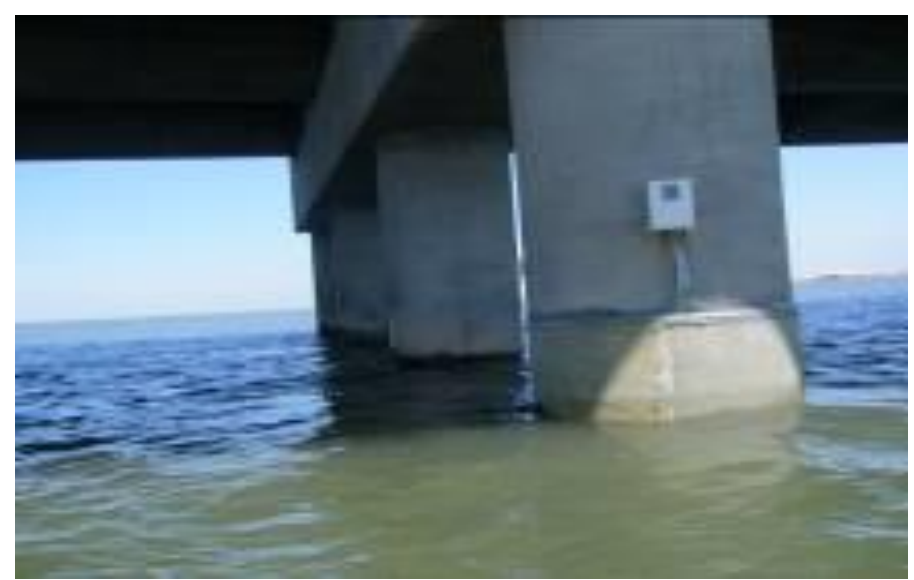

Figura 5. Protecção com fibra de vidro de pilares 


\subsection{Inspecção e manutenção}

O plano de inspecção e manutenção define os locais de inspecção, a periodicidade das inspecções, as anomalias a detectar e os procedimentos de manutenção. As inspecções são baseadas em inspecções visuais, complementadas com algumas medições de modo a detectar

eventuais anomalias (Figura 4). Dadas as características da Ponte Vasco da Gama foram consideradas neste plano algumas situações particulares com sejam o controle das infra-escavção e o retensionamento periódico dos cabos.

\section{REFERÊNCIAS}

Branco, F., Machado, L. (1999), Search for Quality in the Vasco da Gama Bridge. Structures for the Future - The Search for Quality, International Association for Bridge and Structural Engineering - IABSE. Conf. Report. Vol.83. Zurich.

Branco, F., Mendes, P., Guerreiro, L. (2000), Special Studies for The Vasco da Gama Bridge, Journal of Bridge Engineering, ASCE - American Society of Civil Engineering, vol.5, n⿳0 3 , pp.224-232, Reston.

Branco, F. A., Brito, J. (2004), Handbook of Concrete Bridge Management, Ed. American Society of Civil Engineers, 468pp., Reston.

ENV 1998-1-4 (1995), Eurocode 8: Design Provisions for earthquake Resistance of Structures, Part 1-4: Strengthening and Repair of Buildings, European Committee for Standardisation-CEN, Brussels.

ENV 1991-2-4 (1999), Eurocode 1: Basis of Design and Actions on Structures, Part2-4: Wind Actions, European Committee for Standardisation-CEN, Brussels.

Mangat, P., Molloy, B. (1994), Prediction of Long Term Chloride Concentration in Concrete, Material and Structures, Vol.27, pp.338-346. Paris.

Monteiro, I. (2010), Modelação da evolução da carbonatação em elementos de betão armado, Tese de Mestrado, IST, Lisboa

NP EN206 (2007), Especificações, desempenho, produção e conformidade do betão, IPQ. Lisboa.

TRB (1995), Highway Capacity Manual, Special Report No 209. Transportation Research Board. Washington. 